\title{
The lightness of a transparent surface in Metelli's model of phenomenal transparency
}

\author{
SERGIO CESARE MASIN \\ University of Padua, Padua, Italy
}

\begin{abstract}
Metelli's model of phenomenal transparency contends that the properties of an achromatic transparent surface are a function of the lightnesses of the isolated surfaces that are perceived when the region of the field where transparency occurs is viewed with an analytical attitude. It has been shown that when an appropriate hypothesis is introduced as to the condition for the balanced transparency, Metelli's model predicts that the lightness of a transparent surface is the arithmetic mean of the lightnesses of the parts of that surface viewed in isolation. The results of the experiment reported here confirmed the prediction. The results also allowed the derivation of new equations for unbalanced transparency.
\end{abstract}

Achromatic transparent surfaces are essentially characterized by two properties: density and lightness. Metelli (1985) showed that the properties of a transparent surface are a function of the lightnesses viewed in the surface with an analytical attitude and viewed around the part of the visual field where transparency may take place.

Let Figure 1 depict a rectangular filter over a bicolored background. A and B are the background surfaces, and $P$ and $Q$ are the parts-viewed with an analytical attitude-of the filter over A and B, respectively. Let $a$, $b, p$, and $q$ be the lightnesses of $\mathrm{A}, \mathrm{B}, \mathrm{P}$, and $\mathrm{Q}$, respectively. Metelli's model states that

$$
\begin{aligned}
& p=\alpha_{1} a+\left(1-\alpha_{1}\right) t_{1} \\
& q=\alpha_{2} b+\left(1-\alpha_{2}\right) t_{2},
\end{aligned}
$$

where $\alpha_{1}$ and $\alpha_{2}$ were proved to be coefficients expressing the degree of transparency (the property that is the inverse of density) over $\mathrm{A}$ and $\mathrm{B}$; and $t_{1}$ and $t_{2}$ are the lightnesses of the parts of the filter over A and B, respectively.

In Metelli's model, $t_{1}$ is assumed equal to $t_{2}$. Therefore, the model becomes

$$
\begin{aligned}
& p=\alpha_{1} a+\left(1-\alpha_{1}\right) t \\
& q=\alpha_{2} b+\left(1-\alpha_{2}\right) t,
\end{aligned}
$$

which Metelli considered as a system of two equations with three unknowns, that is $\alpha_{1}, \alpha_{2}$, and $t$, where $t$ is the lightness of the entire filter. In order to make it solvable, Metelli assumed that $\alpha_{1}=\alpha_{2}=\alpha$ and automatically restricted the solution only to cases of balanced transparency. His solution is:

I am now affiliated with the University of Udine, Udine, Italy. The CNR supported this paper through a contribution for a 1-month stay at the University of Oregon. I wish to thank F. Metelli and N. Pastore for useful comments. Correspondence may be addressed to me at Dipartimento di Psicologia Generale, Piazza Capitaniato 3, 35135-Padova, Italy.

$$
\begin{aligned}
\alpha & =(p-q) /(a-b) \\
t & =(a q-p b) /(a-p+q-b) .
\end{aligned}
$$

Masin and Gardonio (1985) have found experimentally that $\alpha_{1}$ and $\alpha_{2}$ depend on the differences $a-p$ and $q-b$ (Figure 1). That is, $\alpha_{1}$ appears less than $\alpha_{2}$ when $a-p>$ $q-b$, and $\alpha_{1}$ appears greater than $\alpha_{2}$ when $a-p<q-b$. This leads me to suggest as a plausible hypothesis that the equality $|a-p|=|q-b|$ is the condition for balanced transparency.

Equation 4 may be rewritten in the form of a weighted average, that is

$$
t=u p+(1-u) q
$$

where

$$
u=\frac{q-b}{a-p+q-b} .
$$

The hypothesized condition for balanced transparency, $|a-p|=|q-b|$, implies that $u=1 / 2$. The following experiment was made in order to check the prediction that $t=(p+q) / 2$.

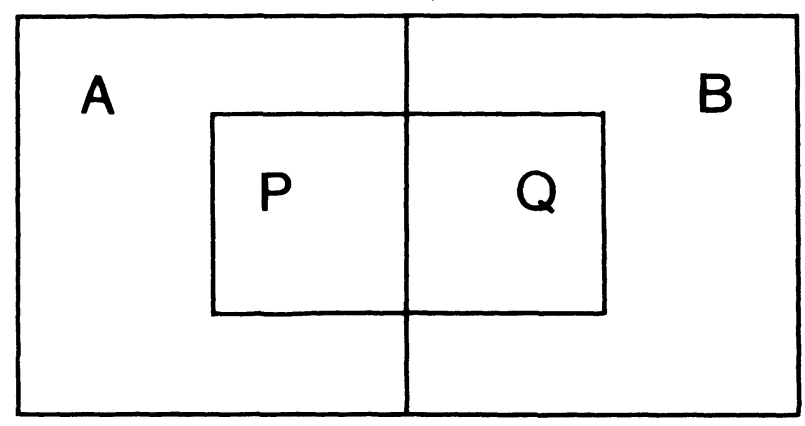

a

Figure 1. Outline representation of an experimental pattern, where a rectangle was perceived as transparent over a bicolored background. 
Table 1

Reflectances for Surfaces A, B, P, and Q (Figure 1), and Reflectance (R) and Transmittance (T) of the Episcotister Simulated in the Experimental Pattern

\begin{tabular}{cllllll}
\hline $\begin{array}{c}\text { Pattern } \\
\text { Number }\end{array}$ & A & B & P & Q & R & T \\
\hline 1 & .40 & .11 & .67 & .52 & .96 & .52 \\
2 & .40 & .11 & .46 & .31 & .52 & .52 \\
3 & .40 & .11 & .31 & .16 & .21 & .52 \\
4 & .40 & .11 & .23 & .08 & .05 & .51 \\
5 & .87 & .02 & .76 & .31 & .64 & .53 \\
6 & .87 & .02 & .67 & .23 & .45 & .52 \\
7 & .87 & .02 & .59 & .16 & .30 & .51 \\
8 & .87 & .02 & .52 & .08 & .15 & .51 \\
9 & .87 & .02 & .46 & .04 & .06 & .50 \\
\hline
\end{tabular}

\section{METHOD}

\section{Observers}

The observers were 40 unpaid university students who were recruited as they entered the department.

\section{Stimuli}

Nine patterns such as that outlined in Figure 1 were used. The side length of squares $A$ and $B$ was $\mathbf{4 0} \mathrm{mm}$, and that of squares $P$ and $Q$ was $22 \mathrm{~mm}$. Each pattern was stuck at the center of a $200 \times 200 \mathrm{~mm}$ gray square with reflectance of .23 . Table 1 lists the reflectances of $A$, B, P, and Q for each pattern. Gray papers from the set of 19 supplied by the NCS Institute (Sweden) were used.

As shown in Table 1, Patterns 1-4 shared the same reflectance for surfaces A and B, as did Patterns 5-9. The retinal stimulation produced by an episcotister rotating at fusion speed in front of surfaces $A$ and B may be simulated by choosing appropriate reflectances for surfaces $P$ and $Q$. These reflectances were chosen to simulate an episcotister with transmittance .5 and with reflectance increasing from Pattern 1 to 4 , and from Pattern 5 to 9. Table 1 also lists these simulated transmittances and reflectances. Perceptually, the transparent rectangle in a pattern became progressively darker in passing from Pattern 1 to 4 , and from Pattern 5 to 9 .

The observer sat at a table provided with a head- and chinrest to keep the eyes level with the pattern. On the same table, the pattern was placed $1.3 \mathrm{~m}$ from the subject on the frontoparallel plane. The pattern, which was viewed binocularly, was shown by removing a white screen. The illumination level was of about $30 \mathrm{~lx}$.

\section{Procedure}

The observer rated the lightnesses of parts A, B, P, and Q (Figure 1) using the numbers in the range of $0-100$. Zero was defined as the whitest white and 100 as the blackest black ever experienced by the observer. Numbers lower than 50 were attributed in proportion to light grays, and numbers higher than $\mathbf{5 0}$ in proportion to dark grays. Subjects were told to call out the numerical response within approximately $5 \mathrm{sec}$. Before starting the experiment, observers were shown all nine patterns in the order 1 to 9 so that they realized the range of variation of grays.

This procedure was common to all 40 observers, which were subdivided into two groups of $20, \mathrm{X}$ and $\mathrm{Y}$.

Group $X$. In addition to rating the lightnesses of A, B, P, and Q, the observers also rated the lightness, $t$, of the whole transparent rectangle. They were instructed to rate the lightness of the transparent rectangle while they perceived it as a unitary transparent strip or rectangular veil; they were told not to heed surfaces $P$ and $Q$, and were urged not to make any sort of valuation of the lightnesses of $P$ and $Q$. Observers rated the lightness of the transparent rectangle with no noticeable hesitation and, when asked, most of them declared that they found no special difficulty in following the instruction.

Observers had to rate $a, b, p, q$, and $t$ in intermixed order, to ward off possible changes in unit (Anderson, 1982, p. 282). There were two sequences of 31 presentations of the patterns. A sequence consisted of
3 presentations of each of the nine patterns. For each of these presentations of a pattern, the observer had to rate the lightness of $P$, of $Q$, or of the whole transparent rectangle. Interspersed, there were 4 more presentations, for each of which the observer was asked to rate the lightness of A or B in one of Patterns 1 to 4, or the lightness of A or B in one of Patterns 5 to 9. (The choice of one of Patterns 1 to 4 , or one of 5 to 9, was at random.) The order of presentation of Patterns 1 to 9 and the choice for the rating of $\mathrm{A}, \mathrm{B}, \mathrm{P}, \mathrm{Q}$, or the whole transparent rectangle for a given pattern were predetermined at random by a microcomputer and were different for each sequence and for each observer. The number of the pattern and the name of the surface was output by the microcomputer, and the experimenter called out the name of the surface to be rated (A, B, P, Q, or the whole transparent rectangle). The observer interpreted the name of a surface by referring to a copy of Figure 1 displayed beneath the experimental pattern.

Group Y. The procedure for Group Y was the same as that for Group $\mathrm{X}$, except for the following changes. In place of the instruction to rate the lightness of the whole transparent rectangle, observers were asked to rate the average lightness of surfaces $P$ and $Q$. Observers were given the instruction, which was followed by all of them, to produce a single numerical response when the task was to rate the average lightness of surfaces $P$ and $Q$, instead of mentally computing the arithmetic mean of two numerical responses, one for surface $P$ and one for surface Q. Instead of calling out "the whole transparent rectangle," the experimenter called out "the average of $p$ and $q$." The random orders of the sequences of presentations were different from those used for Group X.

\section{RESULTS}

The results for Groups $\mathrm{X}$ and $\mathrm{Y}$ are reported in Tables 2 and 3, respectively. For our purposes, a "rough" statistical test is sufficient. Multiple $z$ tests showed that the arithmetic mean of the individual differences, $t-[(p+q) / 2]$ and $m-[(p+q) / 2]$, marked by an asterisk or a dagger, were statistically different from zero, evidencing that $t$ and $m$ barely deviated from $(p+q) / 2$. The arithmetic mean of the individual differences of $t$ and $m$ from the geometric mean of $p$ and $q$ are reported in Column 8 of Tables 2 and 3, respectively. Multiple $z$ tests showed that none of these mean differences in Table 2 were statistically different from zero. A dagger marks the only one of these differences in Table 3 that was statistically different from zero.

A comparison of the mean $p s, q s$, and $t \mathrm{~s}$ in Table 2 with the corresponding mean $p s, q \mathrm{~s}$, and $m \mathrm{~s}$ in Table 3 shows

Table 2

Mean Ratings of $a, b, p, q$, and $t$ from Group $X$

\begin{tabular}{cccccccr}
\hline Pattern & & & & & & $t-\frac{p+q}{2}$ & $t-\sqrt{p q}$ \\
Number & $a$ & $b$ & $p$ & $q$ & $t$ & 0.9 & 1.1 \\
\hline 1 & 37.6 & 84.6 & 22.4 & 27.3 & 25.7 & 0.6 & 1.5 \\
2 & 37.6 & 84.6 & 31.1 & 45.0 & 38.7 & 0.6 \\
3 & 37.6 & 84.6 & 46.0 & 62.7 & 55.3 & 0.9 & 1.8 \\
4 & 37.6 & 84.6 & 55.3 & 85.8 & 63.9 & $-6.7^{*}$ & -4.6 \\
5 & 4.2 & 98.2 & 18.5 & 41.4 & 25.4 & $-4.9 \dagger$ & -2.4 \\
6 & 4.2 & 98.2 & 24.3 & 43.6 & 34.0 & 0.0 & 1.8 \\
7 & 4.2 & 98.2 & 27.1 & 57.2 & 39.0 & $-3.1^{*}$ & .0 \\
8 & 4.2 & 98.2 & 34.0 & 74.4 & 48.3 & $-5.9 *$ & -1.4 \\
9 & 4.2 & 98.2 & 37.5 & 89.4 & 58.7 & -4.8 & 1.5 \\
\hline
\end{tabular}

Note-The symbols $a, b, p$, and $q$ represent the lightnesses of parts A, $\mathrm{B}, \mathrm{P}$, and $\mathrm{Q}$ in Figure $1 ; t$ represents the lightness of the whole transparent rectangle as estimated by the observer. *Significant at the .05 level. †Significant at the .01 level. 
Table 3

Mean Ratings of $a, b, p, q$, and $m$ from Group $Y$

\begin{tabular}{ccccccccr}
\hline $\begin{array}{c}\text { Pattern } \\
\text { Number }\end{array}$ & $a$ & $b$ & $p$ & $q$ & $m$ & $m-\frac{p+q}{2}$ & $m-\sqrt{p q}$ & $t-m$ \\
\hline 1 & 35.9 & 79.8 & 19.6 & 24.1 & 20.0 & -1.8 & -1.5 & 2.7 \\
2 & 35.9 & 79.8 & 30.4 & 37.6 & 33.2 & -0.8 & -.2 & 1.4 \\
3 & 35.9 & 79.8 & 42.4 & 60.9 & 50.6 & -1.0 & .2 & 1.9 \\
4 & 35.9 & 79.8 & 50.7 & 81.8 & 61.4 & $-4.9^{*}$ & -2.6 & -1.8 \\
5 & 7.2 & 97.6 & 14.6 & 35.5 & 21.7 & $-3.3^{*}$ & -.6 & -1.6 \\
6 & 7.2 & 97.6 & 19.9 & 40.7 & 27.2 & -3.1 & -.7 & 3.1 \\
7 & 7.2 & 97.6 & 22.8 & 49.0 & 33.2 & -2.7 & .3 & -0.4 \\
8 & 7.2 & 97.6 & 30.1 & 68.8 & 43.8 & $-5.6 *$ & -1.3 & -0.3 \\
9 & 7.2 & 97.6 & 34.2 & 87.0 & 60.4 & -0.2 & $6.5 \dagger$ & 4.6 \\
\hline
\end{tabular}

Note-The symbols $a, b, p$, and $q$ represent the lightnesses of parts A, $\mathrm{B}, \mathrm{P}$, and $\mathrm{Q}$ in Figure $1 ; m$ represents the average lightness of $p$ and $q$ as estimated by the observer. *Significant at the .05 level. $†$ Significant at the .001 level.

that the means in Table 2 are slightly higher. Reasonably, this is due to a constant, $k$, cuased by interindividual differences in the use of numbers. A meaningful comparison of $t$ and $m$ can be obtained only if we get rid of $k$. That is, $k$ must be added to the $p s$ and $q s$ in Table 3 in order to make them equal statistically to the corresponding $p s$ and $q s$ in Table 2 . And the same $k$ must be added to the $m s$ in Table 3 in order to make them comparable with the $t \mathrm{~s}$ in Table 2 . It may be easily shown that the means $m-[(p+q) / 2]$ in Table 3 are quantities in which the $k$ s added to $m, p$, and $q$ annul each other.

Column 9 of Table 3 reports the differences $t-m$, obtained by subtracting the means $m-[(p+q) / 2]$ from the corresponding means $t-[(p+q) / 2]$. These differences $t-m$ should now be independent of $k$. Multiple $z$ tests showed that none of these differences statistically deviated from zero.

\section{DISCUSSION}

The mean rating of $t$ statistically coincides with the geometric mean of $p$ and $q$, and barely deviates from $(p+q) / 2$. It may be concluded that, approximately, $t=(p+q) / 2$, and that Equation 5 is confirmed. The results reported in Column 9 of Table 3 also confirm that the mean rating of $t$ substantially coincides with the intuitive average of $p$ and $q$.

The results of this experiment prove the empirical relation $t=\sqrt{p q}$ (geometric mean) or, approximately, the theoretical relation $t=(p+q) / 2$ (arithmetic mean), also when $|p-a| \neq|q-b|$ (Tables 2 and 3). These empirical relations, therefore, may serve to derive equations for cases of unbalanced transparency (that is, when $\alpha_{1} \neq \alpha_{2}$ ). Thus, let us go back to Model 2 . Since $t$ is now empirically known, the two equations can be solved independently for both $\alpha_{1}$ and $\alpha_{2}$.

When $t=(p+q) / 2$, the solutions are

$$
\begin{aligned}
& \alpha_{1}=(p-q) /(2 a-p-q) \\
& \alpha_{2}=(q-p) /(2 b-p-q) .
\end{aligned}
$$

When $t=\sqrt{p q}$, the solutions are

$$
\begin{aligned}
& \alpha_{1}=(p-\sqrt{p q}) /(a-\sqrt{p q}) \\
& \alpha_{2}=(p-\sqrt{p q}) /(b-\sqrt{p q}) .
\end{aligned}
$$

Equations 6 and 8 , and Equations 7 and 9, give very similar values of $\alpha_{1}$, and $\alpha_{2}$, respectively, because $(p+q) / 2 \cong \sqrt{p q}$.

Each of Equations 6 to 9, taken individually, shows the properties of a transparency coefficient (like $\alpha$ in Equation 3). When $p=q$ (opacity) then $\alpha=\alpha_{1}=\alpha_{2}=0$. When $p=a$ and $q=b$ (perfect transparency) then $\alpha=\alpha_{1}=\alpha_{2}=1$. When there is some degree of transparency, $\alpha, \alpha_{1}$, and $\alpha_{2}$, assume values between 0 and 1. Equations 6-9 might be considered as the equations for unbalanced transparency that derive from Metelli's model.

\section{REFERENCES}

ANDERSON, N. H. (1982). Methods of information integration theory. New York: Academic Press.

Masin, S. C., \& Gardonio, G. (1985). The valuation of the apparent density of a filter on a bicolored background. Perception \& Psychophysics, 37, 103-108.

Metelli, F. (1985). Stimulation and perception of transparency. Psychological Research, 47, 185-202.

(Manuscript received for publication November 17, 1986.) 